\title{
A time-of-use based electricity price package mechanism in retail market considering consumers' diversity
}

\author{
Gang Luo ${ }^{1}$, Yujun $\mathrm{He}^{2,}$, Chen Zhao ${ }^{1}$, Xuan Zhang ${ }^{1}$, Shaohua $\mathrm{Lin}^{1}$, Yue Zhao ${ }^{1}$, and Yuanji $\mathrm{Cai}^{2}$ \\ ${ }^{1}$ Power Dispatch and Control Centre, Guangdong Power Grid Co. Ltd., Guangzhou 510000, China \\ ${ }^{2}$ State Key Lab of Control and Simulation of Power Systems and Generation Equipments, Dept. of Electrical Engineering, Tsinghua \\ University, Beijing 100084, China
}

\begin{abstract}
In the deregulated retail market, consumers should have more right of choice for paying for their energy demand. This paper focuses on the design of electricity price package mechanism in retail market considering the demand elasticity of consumers. An optimal price package mechanism is proposed to incentivize consumers for peak-clipping/valley-filling. Consumers are able to choose the appropriate one from a set of price packages, each of which consists of specific time-of-use energy price and maximumdemand price. A numerical case study has shown the usefulness and effectiveness of the proposed mechanism.
\end{abstract}

\section{Introduction}

With the worldwide reform of power retail market deregulation, the consumers are becoming more active in the power trading, which leads to a more flexible power system where the demand can be adjusted to follow with the variation of generation. Instead of demand-side management (DSM) by administrative means, nowadays the demand response (DR) by means of dynamic price or incentives is playing an important role to balancing the demand/generation and optimally allocate power system resources [1]. In this context, the utility companies or retailers have to design more appropriate pricing models to attract consumers in the market competition [2].

Many studies have investigated the design of pricing mechanism in retail market. The dynamic price mechanisms, including time-of-use (TOU) pricing and real time pricing (RTP), are proved to solve the shortterm lack of system capacity and improve the economic surplus [3]. The demand elasticity [4-5] and load factors [6-7] at demand side are usually considered by utility companies in designing the retail price. On the basis of demand-price elasticity theory, the work in [8] proposed an optimal retail price model considering the distribution network constraint. In [9], the price assessment method about the user's satisfaction is discussed in terms of energy consumption behaviour of consumers and electricity bills. In [10], a framework for consumer's power demand based on TOU contract characteristics is proposed for minimizing the mean electricity price paid by the customer. In [11], a dynamic multi-objective optimization of TOU price under multi-model structure is investigated. However, the price package mechanism based on TOU price is rarely studied considering detailed models of consumers' behaviour.
This paper focuses on designing an appropriate price package mechanism considering consumers' diversity. Both the load factors and demand elasticity functions are considered to establish a detailed model to describe the behaviour of different consumers with the given price packages. A two-settlement framework for each price package is adopted, including TOU energy price and capacity price related to maximum demand of consumer. The optimization is formulated considering the behaviour of different consumers with the proposed price packages. The rest part of this paper is organized as follows: Section 2 presents the clustering approach for consumers in retail market. The TOU based electricity package mechanism are introduced in Section 3. Then, the optimization of electricity package is formulated in Section 4 and a numerical case study is conducted in Section 5. Finally, Section 6 gives brief conclusions about the proposed mechanism.

\section{Clustering approach for consumers in retail market}

\subsection{Classification of consumers}

Electricity consumers can be classified in different manners based on their position, size or nature of use. The utility company usually apply different tariffs for the different consumers. In this paper, we classify the consumers using their patterns of consumption, including load factors and demand elasticity factors. Specifically, data dimension reduction technology is adopted for a context of TOU with periods of peak hours $(\mathrm{PH})$, off-peak hours $(\mathrm{OH})$ and valley hours $(\mathrm{VH})$.

\footnotetext{
* Corresponding author: yujunhe@mail.tsinghua.edu.cn
} 


\subsubsection{Load factors}

Since the load curve of consumers is too complicated for the clustering analysis, a data dimension treatment is needed. The dimension of load curve can be reduced by adopting a series of load factor parameters based on TOU. The load factor parameters are shown in Table. 1.

Table 1. Parameters in the optimization ( $\mathrm{m}=$ monthly)

\begin{tabular}{|c|c|}
\hline Parameter & Math. Expression \\
\hline $\begin{array}{c}\text { Daily load } \\
\text { factor }\end{array}$ & $\phi_{i}=P_{a v, i} / P_{\text {max }, i}$ \\
\hline $\begin{array}{c}\text { Load factor } \\
\text { of PH }\end{array}$ & $\phi_{i}^{P H}=P_{a v, i}^{P H} / P_{a v, i}$ \\
\hline $\begin{array}{c}\text { Load factor } \\
\text { of OH }\end{array}$ & $\phi_{i}^{O H}=P_{a v, i}^{O H} / P_{a v, i}$ \\
\hline $\begin{array}{c}\text { Load factor } \\
\text { of } \mathrm{VH}\end{array}$ & $\phi_{i}^{V H}=P_{a v, i}^{V H} / P_{a v, i}$ \\
\hline
\end{tabular}

\subsubsection{Demand elasticity}

The demand elasticity reflects the responsiveness of consumers to the variation of price. These patterns depend on the load flexibility, users' habit and their willingness to provide demand response. The demand elasticity factors of consumers can be expressed as the ratio of load variation divided by the ratio of price variation during the intra-hour or inter-hours, respectively shown in (1) and (2).

$$
\begin{gathered}
E_{i, t-t}=\frac{\Delta Q_{i, t}}{\Delta \lambda_{t}} \cdot \frac{\lambda_{t}}{Q_{i, t}} \quad t \in T \\
E_{i, t-t^{\prime}}=\frac{\Delta Q_{i, t}}{\Delta \lambda_{t^{\prime}}} \cdot \frac{\lambda_{t^{\prime}}}{Q_{i, t}} \quad t, t^{\prime} \in T \& t^{\prime} \neq t
\end{gathered}
$$

where $E_{i, t-t}$ is the intra-hour demand elasticity factor at hour $\mathrm{t}$ of consumer $i, E_{i, t-t^{\prime}}$ is the inter-hour demand elasticity factor at hour $t$ in response to the price variation at hour $t^{\prime} \cdot \lambda_{t}$ and $\Delta \lambda_{t}$ are respectively the initial price at $\mathrm{t}$ hour and its variation, $Q_{i, t}$ and $\Delta Q_{i, t}$ are respectively the initial demand and its variation at hour t.

\subsection{Clustering of consumers}

In this work, we adopt K-means method to for clustering consumers. K-means clustering aims to partition $\mathrm{n}$ observations into $\mathrm{k}$ clusters in which each observation belongs to the cluster with the nearest mean, serving as a prototype of the cluster. Given the vector of consumer i is $\mathbf{x}_{i}=\left[\phi_{i}, \phi_{i}^{P H}, \phi_{i}^{O H}, \phi_{i}^{V H}, E_{i, x H-x H}\right]$ and a set of consumers $\left(\mathbf{x}_{1}, \mathbf{x}_{2}, \ldots \mathbf{x}_{i}, \ldots, \mathbf{x}_{N}\right)$, The K-means method will compute $\mathrm{I}(\leqslant \mathrm{N})$ sets $\mathrm{S}=\{\mathrm{S} 1, \mathrm{~S} 2, \ldots, \mathrm{SI}\}$ so as to minimize the within-cluster sum of squares (WCSS). A standard K-means algorithm is based on the iterative process: the first step is to assign each consumer to the cluster whose mean has the least squared Euclidean distance $\left\|\mathbf{x}_{i}-m_{p}\right\|^{2}$, where $m_{p}$ is the centroid of the cluster $p$; then $m_{p}$ is updated using the consumers in the cluster.

$$
m_{p}=\frac{1}{\left|S_{p}\right|} \sum_{x_{i} \in S_{p}} x_{i}
$$

\section{TOU-based electricity price package mechanism}

The TOU pricing mechanism is a typical time-varying tariff differentiated during different periods. TOU prices encourage consumers to shift the use of electricity towards low-demand periods so that the capacity infrastructures in the power system can be fully used by reducing the power gap between peak and valley. The TOU is a sort of differentially pricing method for time domain, whereas it is identical for all consumers. The TOU prices are shown in (4), where $\lambda_{P H}, \lambda_{O H}$ and $\lambda_{V H}$ are respectively the price during $\mathrm{PH}, \mathrm{OH}$ and $\mathrm{VH}$.

$$
\lambda_{t}^{T O U}= \begin{cases}\lambda_{P H} & t \in P H \\ \lambda_{O H} & t \in O H \\ \lambda_{V H} & t \in V H\end{cases}
$$

Similarly, the differentially pricing for different users is called electricity price package. By offering different electricity price packages in the retail market, consumers are possible to choose one package that benefits themselves in terms of maximizing their own economic surplus. Besides, this approach can improve the extent of liberalization of the retail market.

A two-settlement pricing mechanism with energy and capacity prices is widely adopted in China's power retail market. In this paper, we propose a TOU based electricity price package design, each of which consists of specific TOU prices and a maximum-demand price to pay for the capacity. Let $\lambda_{j}^{c a p}$ be the maximum-demand price and $\lambda_{j}^{P H}, \lambda_{j}^{O H}, \lambda_{j}^{V H}$ be the TOU prices of price package $\mathrm{j}$ during $\mathrm{PH}, \mathrm{OH}$ and $\mathrm{VH}$. The demand consumption amount of typical consumer $i$ during $\mathrm{PH}$, $\mathrm{OH}$ and $\mathrm{VH}$ are respectively $Q_{i, j}^{x H}=D_{i}^{x H}\left(\lambda_{j}^{x H}\right)$, $x H=P H, O H, V H$. Supposing the monthly demand amount of consumer $i$ is $Q_{i, 0}$. Thus, $Q_{i, j}^{x H}$ can be expressed as: 


$$
Q_{i, j}^{x H}=Q_{i, 0}^{x H} \cdot\left(\begin{array}{c}
k_{i}^{x H}+\frac{E_{i, x H-P H}}{\lambda_{0}^{e}} \lambda_{j}^{P H}+ \\
\frac{E_{i, x H-O H}}{\lambda_{0}^{e}} \lambda_{j}^{O H}+\frac{E_{i, x H-V H}}{\lambda_{0}^{e}} \lambda_{j}^{V H}
\end{array}\right)
$$

where $k_{i}^{x H}=1-E_{i, x H-P H}-E_{i, x H-\mathrm{O} H}-E_{i, x H-\mathrm{V} H}, Q_{i, 0}^{x H}$ is the part of $Q_{i, 0}$ during $x H, \lambda_{0}^{e}$ is the baseline energy price.

Let $T_{x H}$ be the total hours of peak/off-peak/valley periods, $Q_{i, 0}^{x H}$ can be estimated in (6):

$$
Q_{i, 0}^{x H}=\frac{T_{x H} \cdot \phi_{i}^{x H}}{24 h \cdot \phi_{i}} Q_{i, 0}
$$

where $\phi_{i}$ is the daily load factor of consumer $i, \phi_{i}^{x H}$ is the load factor during $x H, x H=P H, O H, V H$.

The maximum demand $P_{i, j}^{\max }$ of consumer $i$ when he chooses the price package $j$ is expressed in (7).

$$
P_{i, j}^{\max }=\frac{Q_{i, j}^{P H}+Q_{i, j}^{O H}+Q_{i, j}^{V H}}{24 h \cdot \phi_{i}}
$$

\section{Optimization formulation for TOU- based price package}

The utility company hopes to maximize its revenue from consumers when designing the price packages. Supposing the total number of consumers is $N$, the number of clusters of consumers is $I$, each consumer is represented by the typical consumer $i$ which is the central element of the cluster that the consumer belongs to. The overall revenue of the utility company is expressed as:

$$
B_{U C}^{\text {total }}=\sum_{i=1}^{I} \omega_{i} N \sum_{j=1}^{K} p_{i}^{j} B_{i}^{j}
$$

where $\omega_{i}$ is the percentage of typical consumer $i$ over $N$ consumers. $p_{i}^{j}$ is the probability that consumer $i$ chooses the price package $j, B_{i}^{j}$ is the payment of consumer $i$ with package $j$ expressed as:

$$
B_{i}^{j}=\sum_{\substack{x H=P H, O H, V H}} \lambda_{j}^{x H} \cdot D_{i}^{x H}\left(\lambda_{j}^{x H}\right)+P_{i, j}^{\max } \lambda_{j}^{c a p}
$$

The overall demand of $N$ consumers during $x H$ is:

$$
\begin{gathered}
Q_{\text {total }}^{x H}=\sum_{i=1}^{I} \omega_{i} N \sum_{j=1}^{K} p_{i}^{j} D_{i}^{x H}\left(\lambda_{j}^{x H}\right), \\
x H \in\{P H, O H, V H\}
\end{gathered}
$$

The energy costs during different periods are impacted by the diversity of power generation. Without loss of generality, we suppose the energy cost during different cost is in a quadratic form, and the operating cost of each price package is $\rho$, the overall cost of the utility company is expressed as:

$$
C_{U C}^{\text {total }}=\sum_{\substack{x H=P H \\
O H, V H}}\left[\begin{array}{l}
a^{x H}\left(Q_{\text {total }}^{x H}\right)^{2}+ \\
b^{x H} Q_{\text {total }}^{x H}+c^{x H}
\end{array}\right]+\rho K
$$

where $a^{x H}, b^{x H}$ and $c^{x H}$ are cost coefficients of $x H$ period.

It is worth noting that, as the utility company is supposed to provide public service, it should not rise the total energy bill for consumers. Let $\lambda_{0}^{e}$ be the baseline energy price for consumers and $\lambda_{0}^{c a p}$ be the baseline maximum-demand price (we assume all consumers are with unique price). The total revenue of the utility company without price package design can be obtained in (12).

$$
B_{U C}^{n o n}=\sum_{i=1}^{I} \omega_{i} N \cdot\left[\begin{array}{l}
\lambda_{0}^{e} \sum_{\substack{x H=P H \\
O H, V H}} D_{i}^{t}\left(\lambda_{0}^{e}\right)+ \\
\lambda_{0}^{c a p} P_{i}^{\max }\left(\lambda_{0}^{e}\right)
\end{array}\right]
$$

Therefore, we can formulate the optimization problem in which the prices of each package are determined for the objective of maximizing the net profit of utility company while the cost of consumers is not added, as shown in (13):

$$
\begin{aligned}
& \max _{\lambda_{j}^{\text {cap }}, \lambda_{j}^{x H}} f_{U C}^{\text {total }}=B_{U C}^{\text {total }}-C_{U C}^{\text {total }} \\
& \text { s.t. } B_{U C}^{\text {total }} \leq B_{U C}^{\text {non }}, \\
& \lambda_{\min }^{e} \leq \lambda_{j}^{x H} \leq \lambda_{\max }^{e}, \\
& \lambda_{\min }^{\text {cap }} \leq \lambda_{j}^{\text {cap }} \leq \lambda_{\max }^{\text {cap }}, \\
& \forall j \in[1, K], x H=\{P H, O H, V H\}
\end{aligned}
$$

where $\lambda_{\min }^{e}$ and $\lambda_{\max }^{e}$ are the lower/upper bounds of energy price, $\lambda_{\min }^{c a p}$ and $\lambda_{\max }^{c a p}$ are the bounds of maximum-demand price. The hyper-parameter $K$ can be adjusted outside of this optimization.

The entire algorithm of the price package design is shown in Fig 1. Firstly, pre-treatment of consumer data is performed by reducing the data dimension and identifying specific TOU parameters of each consumer; consumers are clustered using K-mean method to obtain 
I-number typical consumers; then, we initialize the optimization conditions and let $\mathrm{K}=1$, by computing the optimization and comparing the net profit of utility company with $\mathrm{K}$ packages and that with $\mathrm{K}-1$ packages, we can find the best $\mathrm{K}$ and the optimal prices in the packages.

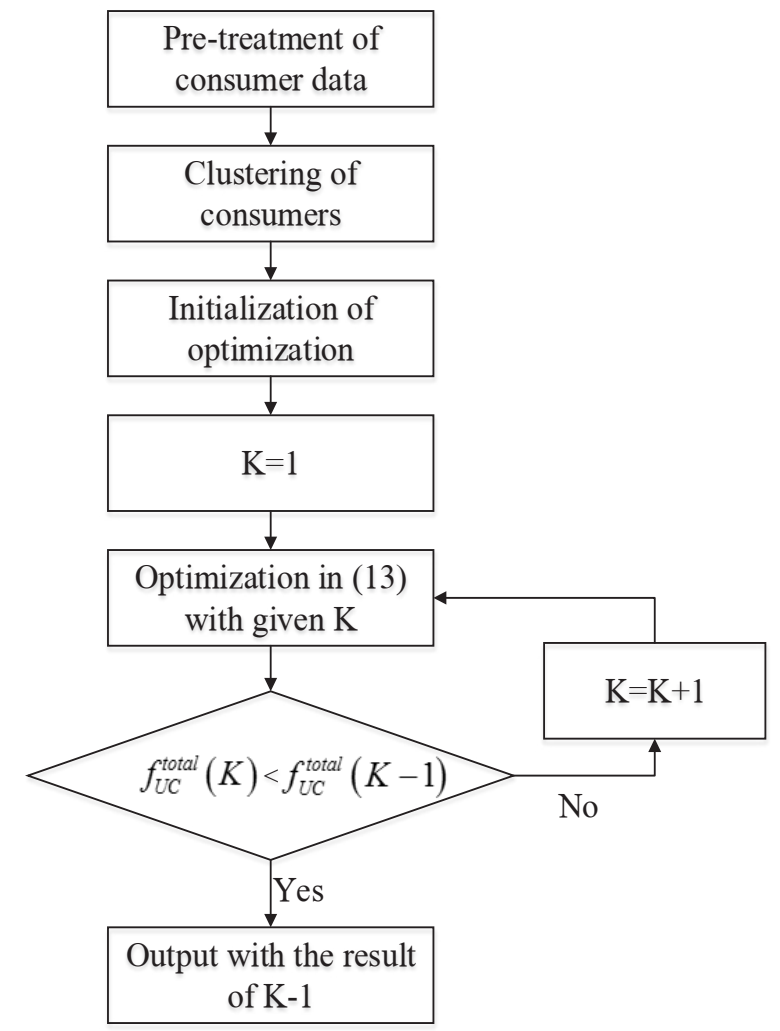

Fig. 1. Algorithm of the price package design.

\section{Case study}

For identifying the proposed method, a numerical case study is conducted in a real scenario with 100 residential consumers. The load curves of consumers are extracted from a database of Guangdong Grid in Southern China. The clustering results of the 100 consumers can be represented by three typical consumers including peakdemand consumer, smoothly-shaped consumer and antipeak consumer, as detailed in follows:

1) Peak-demand consumers, who tend to consume more energy during peak hours even though the price is higher;

2) Smoothly-shaped consumers, who have a smooth load curve with high load factor;

3) Anti-peak consumers, who are willing to fill the valley demand if the price is lower.

The peak-valley TOU periods are set as: 14h00 17h00 and 19h00 22h00 for PH, 0h00 8h00 for $\mathrm{VH}$, and other hours for $\mathrm{OH}$. The costs parameters can be found in [12]. Other parameters for the optimization are shown in Table 2, some of which are based on the Guangdong power market practices.

The optimization is performed using CPLEX12.1 toolbox installed in Matlab r2016b. $K$ is varied from 0 to 4, in which $\mathrm{K}=0$ is the baseline case without price package and prices are not changed, $K=1$ is actually the case of TOU pricing without choice for consumer, $K=2 \sim 4$ means there are some packages optional for consumers. The obtained results are shown in Fig. 2 and Fig. 3. In Fig. 1, the cost of utility company can be reduced when adopting price packages. Fig. 3 shows the net gain from price packages compared with the case $K=$ 0 . It can be observed that the result with $K=3$ price packages in this scenario is best. Fig. 4 shows the contribution of price packages in reducing peak demand and filling valley demand. When $K=2$, the peak demand is reduced at the most, then when $K$ increases, the reduced peak demand is decreased. This is because the price packages give consumers more options if $K$ is greater. The optimal price packages with $K=3$ are shown in Table 3.

Table 2. Parameters in the optimization ( $\mathrm{m}=$ monthly)

\begin{tabular}{|c|r|}
\hline Parameter & \multicolumn{1}{c|}{ Value } \\
\hline$\lambda_{0}^{e}$ & $0.61 Y / \mathrm{kWh}$ \\
\hline$\lambda_{0}^{c a p}$ & $32 Y / \mathrm{kWh} / \mathrm{month}$ \\
\hline$\lambda_{\min }^{e}$ & $0.10 Y / \mathrm{kWh}$ \\
\hline$\lambda_{\max }^{e}$ & $1.2 Y / \mathrm{kWh}$ \\
\hline$\lambda_{\min }^{c a p}$ & $30 Y / \mathrm{kWh} / \mathrm{month}$ \\
\hline$\lambda_{\max }^{c a p}$ & $35 Y / \mathrm{kWh} / \mathrm{month}$ \\
\hline$\rho$ & $1000 Y /$ month \\
\hline
\end{tabular}

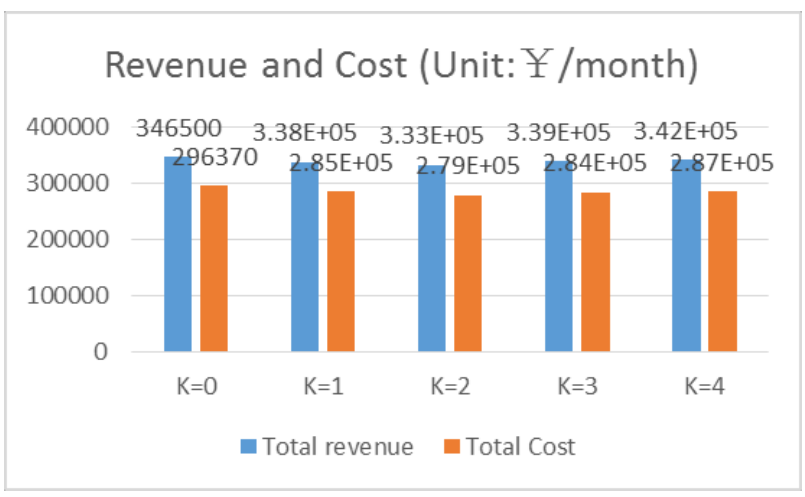

Fig. 2. Revenue and cost with different number of price packages. 


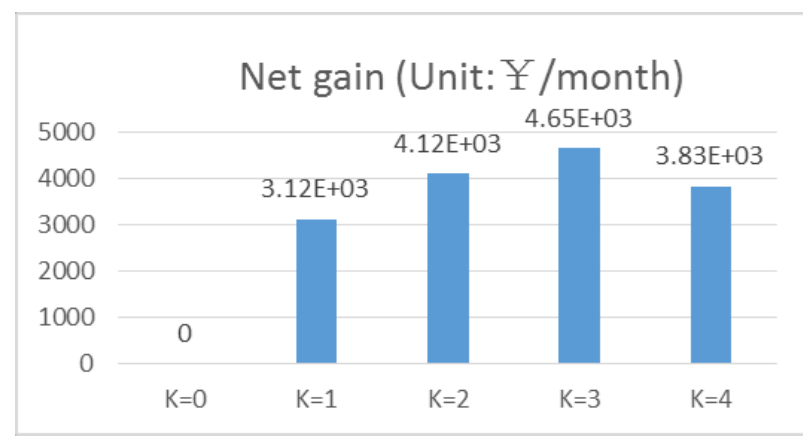

Fig. 3. Net gain with different number of price packages.

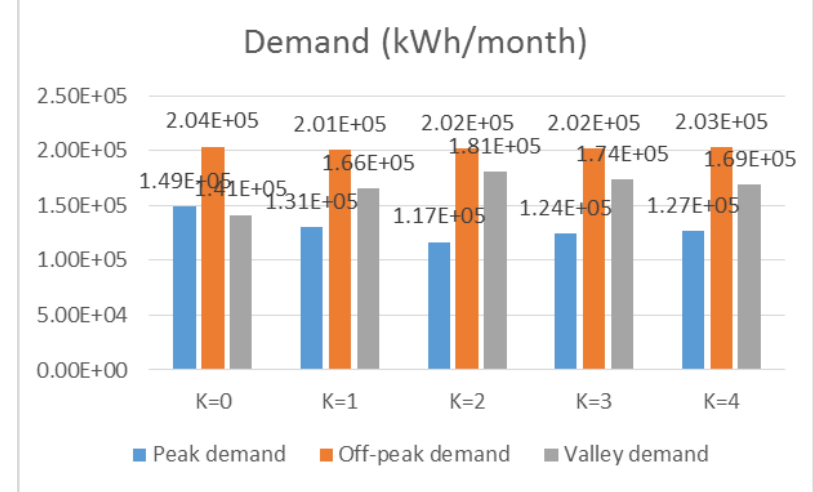

Fig. 4. Demand during different periods with price packages.

Table 3. Optimal price package results when $\mathrm{K}=3$

\begin{tabular}{|c|r|r|r|}
\hline $\begin{array}{c}\text { Price } \\
\text { package No. }\end{array}$ & \multicolumn{1}{|c|}{$\mathbf{1}$} & \multicolumn{1}{|c|}{$\mathbf{2}$} & \multicolumn{1}{|c|}{$\mathbf{3}$} \\
\hline $\begin{array}{c}\lambda_{j}^{P H}(\Psi / \mathrm{kW} \\
\mathrm{h})\end{array}$ & 1.05 & 0.84 & 0.7 \\
\hline $\begin{array}{c}\lambda_{j}^{O H}(Y / \mathrm{kW} \\
\mathrm{h})\end{array}$ & 0.61 & 0.64 & 0.61 \\
\hline $\begin{array}{c}\lambda_{j}^{V H}(Y / \mathrm{kW} \\
\mathrm{h})\end{array}$ & 0.20 & 0.43 & 0.55 \\
\hline $\begin{array}{c}\lambda_{j}^{c a p}(Y / \mathrm{kW} \\
\mathrm{h} / \mathrm{month})\end{array}$ & 35 & 32 & 30 \\
\hline
\end{tabular}

\section{Conclusions}

This paper has investigated a novel TOU price package design mechanism in retail market. The price parameters in the packages are optimized considering the diversity of consumers in terms of load factors and demand elasticity coefficients. The numerical results have shown the effectiveness of the proposed method. With price packages ( $\mathrm{K}$ is greater than 1$)$, the profit of the utility company can be augmented while the consumers are endowed with more right of choice in retail market. Moreover, detailed model with multiple retailers in a competitive environment could be studied in the future work.
This work was supported by Guangdong Power Grid Co., Ltd. Project (No. 036000KK52180031).

\section{References}

1. Y. Bin, S. Kun, L. Dezhi, et al., Research on Power Flexible Load Regulation Technology Based on Demand Response, in proceedings of 2018 8th International Conference on Electronics Information and Emergency Communication (ICEIEC), Beijing, pp. 150-153, (2018)

2. YAO D., CHU Y., Pricing Strategy of Electricity Price Packages under the Power Sale Side Reform in China, Power \& Energy, 39(01): pp.59-61, (2018)

3. R. Li, Z. Wang, S. Le Blond and F. Li, Development of time-of-use price by clustering techniques, in proceedings of 2014 IEEE PES General Meeting, National Harbor, MD, pp. 1-5, (2014)

4. R. Fonteijn, M. Babar and I. G. Kamphuis, An assessment of the influence of demand response on demand elasticity in electricity retail market, in proceedings of 2015 50th International Universities Power Engineering Conference (UPEC), Stoke on Trent, pp. 1-6, (2015)

5. M. Babar, P. H. Nguyen, V. Cuk et al., The development of demand elasticity model for demand response in the retail market environment, in proceedings of 2015 IEEE PowerTech, Eindhoven, pp. 1-6, (2015)

6. S. R. Konda, L. K. Panwar, B. K. Panigrahi et al., Investigating the Impact of Load Profile Attributes on Demand Response Exchange, in IEEE Transactions on Industrial Informatics, vol. 14, no. 4, pp. 1382-1391, (2018)

7. A. B. Arani, R. Yousefian, P. Khajavi et al., Load curve charactristics improvement by means of optimal utilization of demand response programs, in proceedings of 2011 10th International Conference on Environment and Electrical Engineering, Rome, pp. 1-5, (2011)

8. K. Feng, H. Zhou, Z. Liu and D. Hu, Retail market pricing design in smart distribution networks considering wholesale market price uncertainty, in proceedings of IECON 2017 - 43rd Annual Conference of the IEEE Industrial Electronics Society, Beijing, pp. 5968-5973, (2017)

9. HUANG Haixin, DENG Li, WEN Feng. Customer Response Behavior Based on Real-Time Pricing, in Electric Power Construction, 37 (2): 63-68, (2016)

10. Y. Hung and G. Michailidis, Modeling and Optimization of Time-of-Use Electricity Pricing Systems, in IEEE Transactions on Smart Grid, vol. 10, no. 4, pp. 4116-4127, (2019)

11. Y. Hu, Y. Li and L. Chen, Multi-Objective Optimization of Time-of-Use Price for Tertiary Industry Based on Generalized Seasonal MultiModel Structure, in IEEE Access, vol. 7, pp. 8923489244, (2019) 
12. X. Zhang, W. Liu, Y. Chen, et al., Electricity Market Design and Operation in Guangdong Power, in proceedings of $15^{\text {th }}$ International Conference on the European Energy Market, Lodz, pp. 1-5, (2018) 